activities of the two genes would be inversely related, with Ela providing a sort of 'flip-flop' control.

The focus on Ela proteins is of particular relevance because they are known to exert a profound effect on cellular metabolism, activating chromosomal genes for both heat shock protein ${ }^{5}$ and $\beta$-tubulin proteins $^{6}$. Ela proteins have also been shown to activate certain other cellular genes in recombinant plasmids ${ }^{7.8}$. In addition, as first reported by Houweling et al. ${ }^{9}$, if primary rodent cells are transfected with cloned Ela genes alone, they become immortalized. The mechanism of immortalization is unkown but may be the result of stimulation by Ela proteins of the same cellular proliferative functions that are stimulated by the proteins for the benefit of the adenovirus in infected cells. Perhaps the Ela proteins are mimicking the action of an unidentified endogenous stimulator of cell growth that is under the strict control of extracelluar growth regulators.

Since the report of amino acid sequence homology between the Ela and myc oncogene proteins ${ }^{10}$ and the finding that either the Ela gene or the $m y c$ gene will complement a ras oncogene in transforming primary cells ${ }^{11,12}$, any newly discovered property of myc or Ela is promptly sought in the other. Current evidence correlates myc gene expression with cellular proliferation, a feature perhaps akin to Ela's immortalization function. Myc production is transiently induced when growth factors stimulate resting fibroblasts or $T$ cells to enter the $G_{1}$ growth state ${ }^{13-15}$. Regulation of the quantity of myc in the cell may also be important to differentiation. Many cell lineages maintain the ability to proliferate until they acquire markers specific for terminal differentiation, whereupon they withdraw from the cell cycle. During in vitro differentiation of the HL60 promyelocytic cell line, the expression of myc declines as the capacity for unlimited cell division is lost and differentiation markers are induced (ref. 16 and refs. therein).

Is there evidence for normal cellular proteins with the properties attributed here to Ela? Once again, a virus gives some useful information. The polyoma virus enhancer, shown by Borrelli et al. to be blocked by $\mathrm{Ela}$, is inactive in undifferentiated embryonal carcinoma (EC) cells, but becomes active upon their differentiation (ref. 17 and refs. therein). Perhaps a protein in the undifferentiated cells represses the polyoma enhancer, as does Ela in HeLa cells, and perhaps tris protein declines in quantity upon cell differentiation. Further evidence comes from the observation that class I major histocompatibility antigens are absent from undifferentiated EC cells but appear upon differentiation (ref. 18 and refs. therein). Provocatively, the Ela gene of the highly oncogenic strain of adenovirus, adenovirus-12, but not that of adenovirus-2, represses these antigens in transformed rodent cells ${ }^{19}$ and Borrelli et al. suggest that their expression relies on an enhancer, which could be the target for adenovirus-12 Ela. Finally, EC cells do seem to have an Ela-like protein, which is indeed lost upon differentiation ${ }^{20}$.

It is tempting to speculate that cellular proteins possessing activator and repressor functions analogous to those of Ela play a role in gene regulation during the growth and differentiation of cells. By inducing a high level of the proteins, a cell might turn on genes required for cell-cycling and repress other genes that are incompatible with proliferation but required in the resting state or after terminal differentiation. Could myc or a related protein provide such regulation? Experiments assessing any role for myc in gene transcription are scant, but there is a hint that, like Ela, myc can activate heat-shock genes ${ }^{21}$. And, as expected for an Ela-like protein, myc does decrease during $\mathrm{EC}$ cell differentiation ${ }^{14}$. Little is known about enhancer control, but the existence of tissue-specific enhancers ${ }^{22,23}$ makes it highly probable that there is regulation during differentiation. Ela's intriguing properties are directing us to fundamental features of the control of genes and, with time, all will be revealed.
1. Khoury, G. \& Gruss, P. Cell 33, 313 (1983)

Borrelli, E., Hen, R. \& Chambon, P. Nature 312, 608 (1984)

. Berk, A. Jee, F Harrison, T., Williams, J. and Sharp. P.A. Cell 17, 935 (1979)

4. Jones, N. \& Shenk, T. Proc, nain. Acad. Sci. U.S.A. 76. 3665 (1979).

5. Kao, H.-T. \& Nevins, J. Mol. Cell Biol. 3, 2058 (1983)

6. Stein, R. \& Ziff, E. Mol. Cell Biol. (in the press).

7. Treisman, R., Green, M. \& Maniatis, T. Proc. nain. Acod. Sci. U.S.A. 80, 7428 (1983).

- Creen M Treisman, R Manialis, T. Cell 35, 137 (1983).

9. Houweling, A., Van den Elsen, P.J., \& Van det Eb, A.J. Virology 105, 537 (1980).

10. Ralston, R. \& Bishop, J.M. Nature 306, 803 (1983).

11. Land, H.. Parada, L. \& Weinberg, R. Nalure 304. 59 (1983).

12. Ruley, H.E. Nature 394, 602 (1983).

13. Kelly, K. Cochran, B.H., Stiles, C.D. Leder, P. Cell 35. 603 (1983).

14. Campisi, J., Gray, H.E., Pardee. A.B., Dean, M. \& Sonenshein, G.E. Cell 3.241 (1984)

15. Greenberg, M. \& Ziff, E. Nalure 311, 433 (1984)

16. Reitsma, P.H. et al. Noture 306, 492 (1983).

17. Katinka, M., Yaniv, M., Vasseur, M. \& Blangy, D. Cell 20. 393 (1980).

18. Morello, D. et al. Nature 29, 260 (1982).

19. Schrier, P.I., Bernards, R., Vaessen, R., Houweling, A.\& Van der Eb, A. Nature 305, 771 (1983).

20. Imperiale, M.J., Kao, H.-T., Feldman, L.T., Nevins, J.R. \& Strickland, S. Mol. Cell. Biol. 4.867 (1984).

21. Kingston, R.E., Baldwin, A.S. Sharp, P.A. Nature 312. 280 (1984).

22. Banerii, I. Olson, \& Schaffner, W. Cell 33, 729 (1983). 23. Gillies, S., Morrison, S. \& Tonegawa, S. Cell 33, 717 (1983).

Anna Velcich and Edward Ziff are in the Department of Biochemistry and the Kaplan Cancer Research, New York University Medical Center, NY 10016, USA.

\title{
Low-temperature physics
}

\section{Do cosmic rays account for superfluid ${ }^{3} \mathrm{He}$ transition?}

\section{from P.V.E. McClintock}

IN THEORY, the transition between the two principal superfluid forms of liquid ${ }^{3} \mathrm{He}$ ought not to be able to occur. So why is it routinely observed to take place? A possible answer to this intriguing question has recently been put forward by $A$.J. Leggett. Writing in Physical Review Letters 53, 1096; 1984, he suggests that the transition, although prohibited from occuring spontaneously, readily takes place because of the occasional high energy cosmic ray that passes through any experimental chamber on the Earth's surface.

When helium gas is sufficiently cooled under atmospheric pressure, it eventually - albeit somewhat reluctantly on account of its very weak interatomic forces condenses to form a colourless liquid. The liquid is of exceptional importance and scientific interest, enjoying the unique distinction of remaining a liquid down to the lowest attainable temperatures. There are two distinct forms of tinuid helium, corresponding to the two stable isotopes, ${ }^{3} \mathrm{He}$ and ${ }^{4} \mathrm{He}$. Liquid ${ }^{4} \mathrm{He}$ undergoes a superfluid transition at about $2 \mathrm{~K}$, entering a state in which a component of the liquid loses all its viscosity. It thus becomes able to flow quite effortlessly through holes or pores of vanishingly small dimensions; correspondingly, a small object travelling through the superfluid encounters absolutely no resistance to its forward motion. It requires temperatures about 1,000 times colder for the same sort of behaviour, but in much more complicated form, to be observed in liquid ${ }^{3} \mathrm{He}$.

When liquid ${ }^{3} \mathrm{He}$ is cooled under a pressure of more than about 20 atmospheres in a weak magnetic field, there is a very rapid onset of superfluidity at about $2 \mathrm{mK}$. The transition is second order, with no associated latent heat, and it always occurs at the same characteristic temperature for any given pressure. The superfluid phase that results is known as the A-phase. If the liquid is cooled further, it subsequently undergoes a first order transition, to the so-called B-phase superfluid. This transition is analogous to the more familiar phase changes of boiling or freezing, and occurs at a temperature that is not accurately reproducible. To put it another way, if the liquid is being cooled at a steady rate, the time taken for transition from A to B to occur is different on each occasion even if each experiment is carried out with the same cell and, so far as can be determined, in an identical fashion.

There is worse to come. In any pure system close to a phase transition, one can imagine small regions of opposite phase continually forming and disappearing again as the result of thermal fluctuations. Whether any one such region subsequently grows or shrinks will depend both on its 
size and on the various bulk and surface energies of the two phases. Thus, ${ }^{3} \mathrm{He}-\mathrm{A}$ could be cooled to a temperature where it would 'like' to become ${ }^{3} \mathrm{He}-\mathrm{B}$, because the energy of the latter phase is lower, but cannot until a spontaneous thermal fluctuation produces a 'large enough' region of ${ }^{3} \mathrm{He}-\mathrm{B}$. Large enough, in this context, means that the increase in the surface energy between the two phases, resulting from a small increase in the radius of the bubble of B-phase, is more than balanced by the corresponding decrease in energy due to the enclosed liquid being in the lower energy state: if this criterion is met, then the bubble of B-phase will obviously tend to expand until it has taken over the whole of the sample, whereupon the transition from A to $B$ will be seen to have occured.

It is possible to calculate the critical radius for the bubble of ${ }^{3} \mathrm{He}-\mathrm{B}$ and hence, by the application of statistical mechanics, to estimate the average length of time jefore the transition occurs, given any particular degree of supercooling. Even on the most optimistic assumptions about the numerical values of the various parameters, the calculated result is a period of time vastly in excess of the age of the Universe. Hence, the transition should never be observed. And yet, even with a moderate degree of supercooling, the observed transition time is of the order of minutes.

Leggett's solution to this apparent impasse is based upon a consideration of the events that should follow the transit of a cosmic ray muon of about $2 \mathrm{GeV}$ through the supercooled liquid, as can be expected every few minutes. The immediate consequences are reasonably predictable on the basis of detailed studies of high energy particles in helium bubble chambers: a number of relatively low energy electrons would be produced, each of which would give up much of its kinetic energy through he production of heat, initially creating a region of a few hundred $\AA$ in radius at a few tenths of a $K$, while the bulk of the liquid remained at its ambient temperature of 1-2 $\mathrm{mK}$. What would happen next is a matter of some conjecture. Leggett's view is that an expanding shell of heated liquid would be created, propagating out from each original event, with the enclosed liquid cooling towards its ambient temperature. He points out that this 'baked Alaska' distribution would provide ideal conditions for the formation of B-phase ${ }^{3} \mathrm{He}$ as the heated liquid cools through the superfluid transition, and that the cooling would be too rapid for the expansion and domination of any small bubbles of A-phase ${ }^{3} \mathrm{He}$ that might be nucleated at the same time. From the point of view of the experimenter, unaware of the passage of the initiating cosmic ray, the transition from $A$ to $B$ will seem to have taken place spontaneously, a few minutes after the regime of a supercooled A-phase has been entered.

Now that Leggett's suggestion has been aired, it should not be too difficult to set up suitable particle detectors and a coincidence counting system to test whether the transition coincides with the passage of a cosmic ray through the liquid. If the hypothesis is correct, it will open the way to study the metastable A-phase right down to near-zero temperatures in weak magnetic fields - a matter of considerable experimental and theoretical interest. All that should be necessary is to shield the sample from cosmic rays, perhaps by conducting the experiments at the bottom of a deep mine. The first experimental test of Leggett's hypothesis will be awaited with very considerable interest.

P. V.E McClintock is in the Department of Physics, University of Lancaster, Lancaster $L A 14 Y B, U K$.

\title{
Oncogenic intelligence
}

\section{Cell immortalization and transformation by the $p 53$ gene}

\author{
from D.P. Lane
}

A DIRECT role for the so-called $p 53$ gene of cells in the process of oncogenesis is suggested by three papers in this issue of Nature. Parada et al. ${ }^{1}$ and Eliyahu et al. ${ }^{2}$ both show that the protein encoded by the p53 gene can complement activated ras genes in the transformation of primary rodent cells. The third report, from Jenkins et al. ${ }^{3}$ establishes that the $p 53$ gene can also immortalize such cells.

The $\mathrm{p} 53$ protein was first detected in the form of its tight non-covalent complex with the large $T$ protein of simian virus $\mathbf{4 0}$ (SV40) ${ }^{4}$. Subsequently it was found also to complex to the Elb $58 \mathrm{~K}$ protein of adenovirus ${ }^{5}$. Though not closely related in structure, both these proteins are involved in the oncogenic action of their viruses, implying that their shared ability to bind the $\mathrm{p} 53$ protein is important for their action in cellular transformation. The $\mathrm{p} 53$ protein in primary cells and established non-transformed cells has a very short half-life and is present in minute concentrations, with each cell containing only a few hundred molecules. When bound to either viral protein, the half-life of p53 is greatly extended and the protein accumulates, reaching concentrations of the order of 10,000 molecules per cell. Many transformed cell lines and primary tumour isolates also contain elevated levels of the protein $^{6,7}$. Microinjection of antibodies to p53 into the cell nucleus of normal quiescent cells prevents their stimulation by serum, implying an important natural role for the protein ${ }^{8}$

The new discoveries are provocative because they suggest that the alterations in p53 levels and stability in many mouse and human tumours may be directly involved in their altered growth. Parada et al. introduced a cloned p53 gene (linked to the murine leukaemia virus LTR) into primary rat embryo fibroblasts (REF) and Rat-1 cells. While the $p 53$ gene alone failed to transform either cell type, when it was introduced together with an activated ras gene, foci were produced in the REF cultures. Since the ras gene alone was also unable to induce foci, the $p 53$ gene seems to provide a complementation function in this assay in much the same way as the cellular $m y c$ gene, adenovirus Ela gene and the large $T$ gene of polyoma virus 9.10 . Parada et al. further establish that cells transfected with both $p 53$ and myc genes give rise to tumours in nude mice. In both the focusforming assay and the tumorigenicity test, the $p 53$ gene appears to be less efficient than the myc gene.

Eliyahu et al. obtain broadly similar results with either REF cells or Chinese hamster embryo fibroblasts. Again, p53, like $m y c$, complements an activated ras gene in focus-forming assays. Interestingly, this group had some difficulty in establishing cell lines from the foci resulting from introduction of $p 53$ and ras genes together, and obtained evidence to suggest that overproduction of the p53 protein can be very toxic to the cells.

Jenkins et al. go on to prove that p53 can immortalize cells, thus increasing the strength of the similarity between $p 53, m y c$ and Ela. The Wistar adult xiphisternum chondrocyte cells they used have a doubling-time of greater than 60 hours and undergo 30 doublings in culture before senesence and cessation of growth. But after transfection with a plasmid that contained the p53 gene, early passage cultures of the cells had an extended life (200 doublings so far), a shorter doublingtime in serum, and the ability to be transformed efficiently into tumorigenic cells by an activated ras gene.

Since $p 53$ regulation is altered in so many human tumours, an obvious first priority, now is to look for alterations in the p 53 gene in human neoplasia. But deeper issues can also be addressed. Does SV 40 large T have the unusual ability to transform primary cells both readily and directly because it first immortalizes by stabilizing p53 and then induces morphological transformation by some other ras-like activity? If so, why are Ela proteins, rather than the p53-stabilizing E1b $58 \mathrm{~K}$ protein, the immortalizing proteins of adenovirus? Moreover, why do certain small aminoterminal fragments of SV 40 large $T$ that are unable to complex p53, nevertheless have the ability to immortalize cells ${ }^{\prime \prime}$ ? 